\title{
Internal stress distribution in weld-affected zone under the effect of constrained loads
}

\author{
Nikita V. Hapov ${ }^{1}$, Aigul S. Kulesh ${ }^{1, *}$, and Anton A. Gerasimov ${ }^{1}$ \\ ${ }^{1}$ National Research Tomsk Polytechnic University, 634050 Tomsk, Russia
}

\begin{abstract}
The paper presents the experimental results of identify the redistribution pattern under the influence of mechanic cycle loads. The subject of the research was the weld metal zone of the uniform joint made of steel $\mathrm{X} 12 \mathrm{Cr} 1 \mathrm{MoV}$. The research method was to organize the mechanic cyclic deformation of sample and to increase the load in each regular load cycle. The result of the work is the determination of sign-variable pattern of internal stress changes under deformation leading to propagation of fatigue and destruction.
\end{abstract}

\section{Introduction}

There is a major number of emergencies connected with heat-affected zone fatigue as well as with weld-affected zone. It is proportional to the increase of welded seam use.

Durability of welded seams can be obtained by rational selection of constructional, metallurgical and technological means. Nevertheless, the working capacity and welded seam functional qualities required are not always provide

Among the broad range of tasks solved in the framework of the Russian energy development, among the first in importance is the problem of ensuring the reliability and efficiency of the thermal power plants equipment.

This applies in particular to those components where in manufacture materials different in their mechanical, chemical, strength and thermal characteristics are used. It is obvious that the most dangerous elements in this sense are welded joints which transitional zone is a kind of stress concentrators.

The durability calculation is based on admissible stress in welded seam design. In its turn, admissible stress is based on the material properties, type of effort (stretching, bending, shearing) and type of load. They are determined as a quotient of conventionally disrupting load by factor of safety.

If a welded design works under the repeated loads and it experiences the cycle fatigue and fatigue damage, admissible loads should be directly reduced in relation to factored stress.

However, admissible loads cannot match the actual design stresses by an order of magnitude. It refers to the hard-to-predict internal stress field redistribution in particular. This is what may cause the destruction by itself.

* Corresponding author: ronikul@tpu.ru 
In this regard, the aim of the work was to identify the redistribution pattern under the influence of mechanic cycle loads.

\section{Materials. Samples. Experimental techniques}

The research sample was cut off the pipe of a superheater (Fig. 1) in the brick envelope zone (point B, Fig. 1). The pipes were made of steel X12Cr1Mo) in 1999. The weld assembly was made in 2005 . The pipes in the boiler were operating for 16500 hours. Working temperature in point $\mathrm{B}$ was $480^{\circ} \mathrm{C}$. The pipe was taken out of service due to the fracture in point $\mathrm{A}$, where the flue gas flow temperature was up to $540-590{ }^{\circ} \mathrm{C}$.

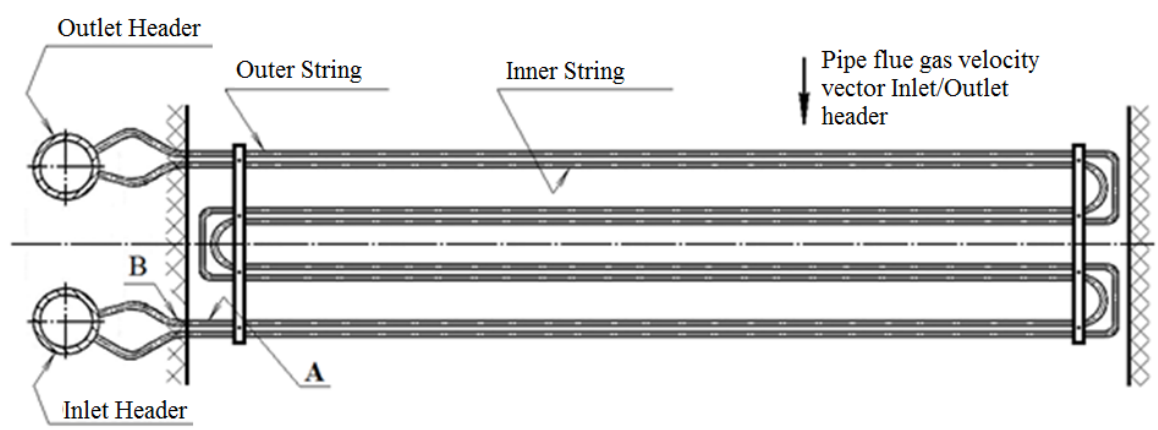

Fig. 1. Top view of the superheater.

The subject of the research was the weld metal zone of the uniform joint made of steel $\mathrm{X} 12 \mathrm{Cr} 1 \mathrm{MoV}$. The research sample was cut off at a distance of 7,5 $\mathrm{mm}$ from the welding joint axis (Fig. 2).

The research method was to organize the mechanic cyclic deformation of sample and to increase the load in each regular load cycle.

The rate of macrostress of the first kind was implemented with the Röntgen's method for the crystal lattice parameter alteration based on Hooke's law.

$$
\frac{\Delta \ell}{\ell}=\frac{\sigma}{\mathrm{E}} \quad \text { or } \quad \frac{\Delta \mathrm{a}}{\mathrm{a}}=\frac{\sigma}{\mathrm{E}},
$$

where $\sigma$ is the internal tensile stress of the first kind, $\Delta \mathrm{a} / \mathrm{a}$ is the relative elongation of lattice cell parameter during the stretching, $\mathrm{E}$ is the Young's modulus.

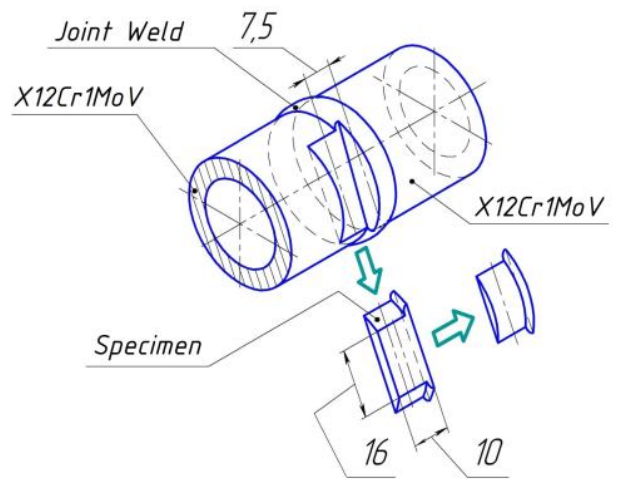

Fig. 2. Sample made of $\mathrm{X} 12 \mathrm{Cr} 1 \mathrm{MoV}$ steel for the research of circumferential zone. 


\section{The experimental facts. Discussion}

As a result of extrenal pulse disturbance application, shear strain tracks can be observed (Fig. 3). Plastic shear band spreads from the mid-point to the edge of the sample, then dissipates on the unevennesses (grains) and goes around them. The waves interfere beyond the unevennesses and transform a developed surface relief into interferential rings shape (Fig. 3a). The deformable sample surface takes a pitting form (Fig. 3b, 3c). The important consistent pattern about the plastic shearing process is the fragmentation and localization tendency at all stages.

The area in front of shear wave is subjected to deformation hardening. At a certain moment microband can't overcome the hardening zone and slows down emerging on the surface as a wave with peak and deepening, determined by material capacity for plastic flow (Fig. 3d). Probably, the shear wave slowing down moment fixates the fluidity limit of the material and is a pre-destruction sign.

Subsequent load application leads to the emergence of individual destructions that are represented as rather big pores (Fig. 4). In the next load cycles separated pores on the sample's surface merge and form main cracks (Fig. 3e).

So, it makes possible to see the interaction of two separate processes at the same time in alternate cycle load conditions. These are creep and fatigue, which lead to the destruction stage. The impact of cycle load on the destruction is the wave periodic deformation hardening known as annealing.

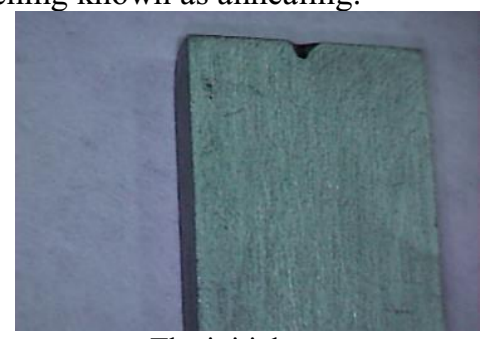

The initial state

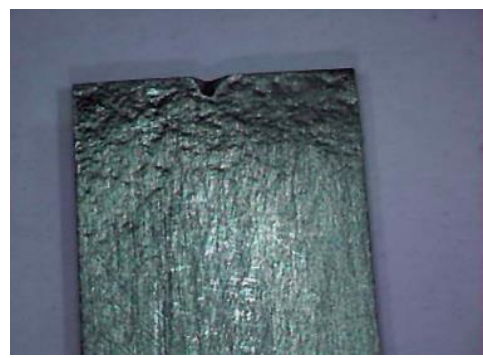

b - $320 \mathrm{MPa}$

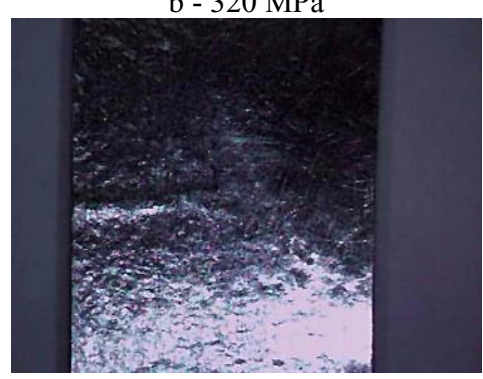

$\mathrm{d}-470 \mathrm{MPa}$

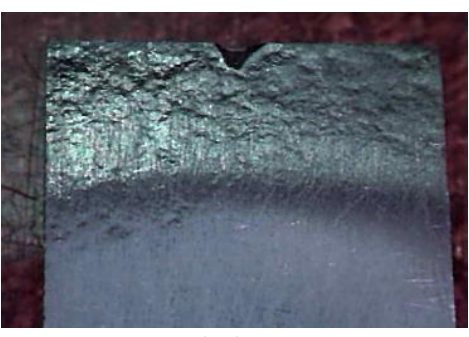

$\mathrm{a}-270 \mathrm{MPa}$

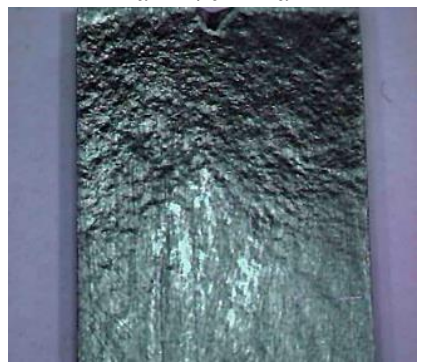

c- $360 \mathrm{MPa}$

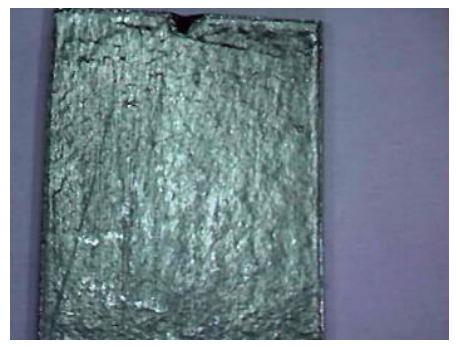

e - 570MPa

Fig. 3. Testing surfaces of the sample. 


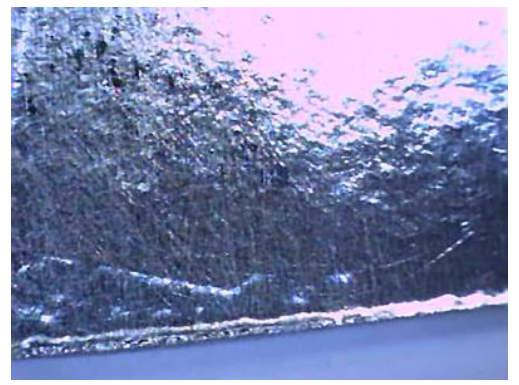

Fig. 4. Porosity and Crack formation of the sample.

It is verified by the results of cyclic internal stress changes. According to Fig. 5 there are two types of interacting processes run coherently in deformation section. On one hand, these are relaxation processes that emerge as a result of dislocation movements, i.e. plastic relaxation forming. On the other hand, every plasticity act is accompanied by acoustic wave generation, which is connected with elastic deformation redistributing during the relaxation acts of deformation.

Internal stress relaxation is a typical process during deformation (Fig. 5). It matches the annealing and marks the forming moment of microstructurally short cracks [1-5]. Interatomic bonds are strained in the grain boundaries. And atomic bonds will break when the interatomic distance increases by more than $10-15 \%$ [1]. The bond breaking process is the leading process at all stages of destruction. Its result is the stress relaxation and crack forming [1-5].

According to A. Griffith's notion, the crack disclosure critical stress for plane strain is written as [6]:

$$
\sigma=\sqrt{\frac{2 E \cdot \gamma}{\pi \cdot(1-\mu) \cdot l}}
$$

where $\mathrm{E}$ is the Young's modulus, 1 is the crack's length, $\mu$ is the Poisson's ratio, $\gamma$ is the free surface energy density. $\left(\gamma=0,1 \mathrm{E} \cdot \mathrm{b} ; \mathrm{b}=2,48 \cdot 10^{-8} \mathrm{~cm}\right.$ is the interatomic distance).

It's on this basis that the length of implied crack can be evaluated with Fig. 5a,b. When deep (zero) relaxation occurs crack length under load of $\mathrm{P}=280 \mathrm{MPa}$ (Fig. 5) will be:

$$
l=\frac{2 E \cdot \gamma}{\pi \cdot(1-\mu) \cdot \sigma^{2}}=\frac{2 \cdot\left(205 \cdot 10^{3} \cdot 0.1 \cdot 205 \cdot 10^{3} \cdot 2.48 \cdot 10^{-8}\right)}{\pi \cdot(1-0.27) \cdot 280^{2}}=1.16 \cdot 10^{-3} \mathrm{~cm} \approx 12 \mu \mathrm{m} .
$$

In this case, internal pipe surface relaxation occurs earlier than the external one (75 $\mathrm{MPa}$ ), and the implied crack length is:

$$
l=\frac{2 E \cdot \gamma}{\pi \cdot(1-\mu) \cdot \sigma^{2}}=\frac{2 \cdot\left(205 \cdot 10^{3} \cdot 0.1 \cdot 205 \cdot 10^{3} \cdot 2.48 \cdot 10^{-8}\right)}{\pi \cdot(1-0.27) \cdot 75^{2}}=16.16 \cdot 10^{-3} \mathrm{~cm} \approx 162 \mu \mathrm{m} .
$$




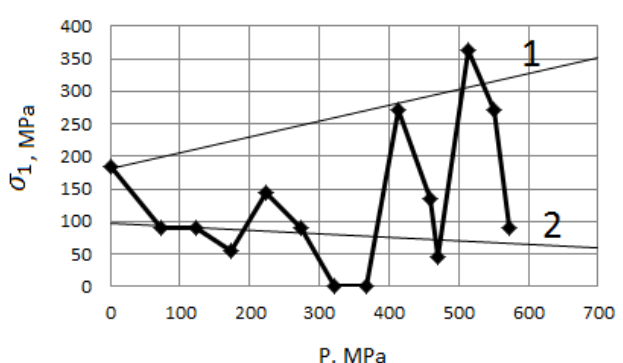

(a)

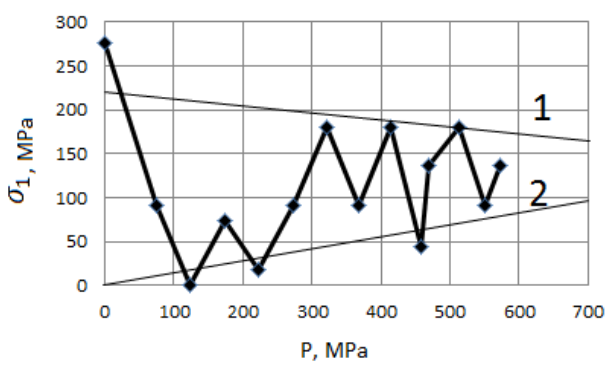

(b)

Fig. 5. Distribution of internal stresses in circumferential zone of joint weld: $a-$ external surface; $b$ internal surface (1 - hardening line; 2 - annealing line).

\section{Conclusion}

The result of the work is the derived plastic flow localization tendency, which starts up with the plastic flow and ends up with the fusion of macroscopical plastic localization areas in the moment of destruction. It enables us to determine propagation features of plastic deformation localization during the material pre-destruction stage.

Another result is the determination of sign-variable pattern of internal stress changes under deformation leading to propagation of fatigue and destruction.

This work was supported by RFBR grant "Fundamentals of engineering sciences" No. 15-08-99544a in 2014, No. 18-08-01265 in 2018).

\section{References}

1. J. Friedel, B. D. Gullity, C. Crussard, Acta Met 1, 79 (1953)

2. L. L. Lyubimova, A. A. Tashlykov, R. B. Tabakaev, A. A. Levin, A. Y. Popov Engineering Failure Analysis 81, 45 (2017)

3. L. L. Lyubimova, R. N. Fisenko, A. A. Tashlykov, R. B. Tabakaev, Met. Mater. Int. 24, 121 (2018)

4. L. L. Lyubimova, R. N. Fisenko, R. B. Tabakaev, A. A. Tashlykov, A. S. Zavorin, Mater. Sci. Eng. A 682, 248 (2017)

5. L. L. Lyubimova, R. B. Tabakaev, A. A. Tashlykov, A. S. Zavorin, V. Y. Zyubanov, EPJ Web Conf. 110, 1037 (2016)

6. A. A. Griffith, Phil. Trans. Roy. Soc. London A 221, 163 (1920) 